\title{
Exponential Mean Square Stability of the BE Method for Linear Neutral Hybrid Stochastic Delay Differential Equations
}

\author{
Haiyan Yuan ${ }^{1,2 *}$, Jihong Shen ${ }^{1}$ \\ ${ }^{1}$ College of Automation, Harbin Engineering University, Harbin, China. \\ ${ }^{2}$ Department of Mathematics, Heilongjiang Institute of Technology, Harbin, China. \\ * Corresponding author. Tel.: +8615045061030; email: yhy82_47@163.com \\ Manuscript submitted March 10, 2014; accepted June 7, 2014. \\ doi: 10.17706/ijapm.2016.6.3.104-111
}

\begin{abstract}
In this paper, the backward Euler (BE) method is introduced and analyzed for linear neutral hybrid stochastic delay differential equations. The exponential mean square stability of the BE method is considered for linear neutral hybrid stochastic delay differential equations. It is proved that, under the one-sided Lipschitz condition and the linear growth condition, for some positive stepsizes which depend on the Markovian switching, the BE method is exponential mean square stable. A numerical example is provided to illustrate the theoretical results.
\end{abstract}

Key words: Linear neutral hybrid stochastic delay differential equations, exponential mean square stability, BE method, Markovian switching.

\section{Introduction}

The practical systems which experience abrupt changes in their structure and parameters caused by phenomena, such as component failures or repairs, changing subsystem interconnections, and abrupt environmental disturbances must be modeled by the hybrid systems driven by continuous-time Markov chains [1]. The hybrid systems combine a part of the state that takes values continuously and another part of the state that takes discrete values. One of the important classes of hybrid systems is the stochastic delay differential equations (SDDEs) with Markovian switching. The natural extensions of this system are stochastic delay integro-differential equations (SDIDE) with Markovian switching and neutral stochastic delay differential equations (NSDDE) with Markovian switching. Although the theoretical study of stochastic differential delay equations with Markovian switching is considered in [2]-[4], the explicit solutions can hardly be obtained for the SDIDEs and NSDDEs with Markovian switching. Rathinasamy and Balachandran [5] analyzed the numerical MS-stability of second-order Runge-Kutta schemes for multi-dimensional stochastic differential systems with one multiplicative noise, the convergence and stability of the Euler method for a class of linear stochastic differential delay equations with Markovian switching is studied in [6]. The numerical method for SDIDEs with Markovian switching had been studied in [7], mean-square stability of Milstein method was obtained. For the neutral stochastic differential equations, Mao studied the exponential mean square stability of its analytical solution in [8], the delay-dependent exponential stability conditions for linear neutral stochastic differential systems was studied in [9]-[11]. Huang Chengming and Wang Wansheng studied the stability of semi-implicit Euler method and $\theta$-method 
for nonlinear neutral stochastic delay differential equations in [12], [13].

To best of our knowledge, no results on the numerical methods for linear NSDDEs with Markovian switching have been presented in the literatures. Thus, it is necessary to develop appropriate numerical methods and to study the properties of these approximate schemes for NSDDEs with Markovian switching.

In this paper we consider the linear $d$-dimensional neutral stochastic delay differential equation with Markovian switching of the form

$$
\left\{\begin{aligned}
d[x(t)-N(x(t-\tau))]= & {[-A(r(t)) x(t)+B(r(t)) x(t-\tau)] d t } \\
& +[D(r(t)) x(t)+E(r(t)) x(t-\tau)] d w(t), t \geq 0, \\
x(t)=\varphi(t), t \in[-\tau, 0] &
\end{aligned}\right.
$$

where $r(t)$ is a Markov chain taking values in $S=\{1,2, \ldots, N\}$ and $A(\cdot), B(\cdot), C(\cdot), D(\cdot)$ and $E(\cdot) \in \mathrm{R}, \quad N$ is the $d$-dimensional square matrices.

In Section 2, we will introduce some necessary notations and assumptions. In Section 3, the definition of exponential mean squares stability is defined, the BE method will be used to produce the numerical solutions and the main result will be shown and proved in this section. A numerical example is given in Section 4.

\section{Notation and Preliminaries}

Throughout this paper, unless otherwise specified, we use the following notations. If $A$ is a vector or matrix, its transpose is denoted by $A^{\mathrm{T}}$. Let $|\cdot|$ denote both the Euclidean norm in $\mathrm{R}^{d}$ and the trace (or Frobenius) norm in $\mathrm{R}^{d \times d}$ (denoted by trace $|A|=\sqrt{\operatorname{trace}\left(A^{\mathrm{T}} A\right)}$ ). $a \vee b$ represents $\max \{a, b\}$ and $a \wedge b$ denotes $\min \{a, b\}$. Let $(\Omega, \mathrm{F}, \mathrm{P})$ be a complete probability space with a filtration $\left\{\mathrm{F}_{t}\right\}_{t \geq 0}$ satisfying the usual conditions, that is, it is right continuous and increasing while $\mathrm{F}_{0}$ contains all P-null sets. Let $w(t)$ be a d-dimensional Brownian motion defined on this probability space, and $\tau$ is a positive fixed delay.

Let $r(t), t \geq 0$ be a right-continuous Markov chain on the probability space taking values in a finite state space $S=\{1,2, \ldots, N\}$ with generator $\Gamma=\left(\gamma_{i j}\right)_{N \times N}$ given by

$$
\mathrm{P}\{r(t+\Delta)=j \mid r(t)=i\}= \begin{cases}\gamma_{i j} \Delta+0(\Delta) & \text { if } i \neq j, \\ 1+\gamma_{i i}+0(\Delta) & \text { if } i=j\end{cases}
$$

where $\Delta>0$. Here $\gamma_{i j} \geq 0$ is the transition rate from $i$ to $j$ if $i \neq j$ while $\gamma_{i i}=-\sum_{j \neq i} \gamma_{i j}$. We assume that the Markov chain $r(\cdot)$ is independent of the Brownian motion $w(\cdot)$. It is known that almost every sample path of $r(t)$ is a right-continuous step function with a finite number of simple jumps in any finite subinterval of $\mathrm{R}_{+}$. As for $r(t)$, the following lemma is satisfied.

Lemma 2.1 [6]. Given $h>0$, then $\left\{r_{n}^{h}=r(n h), n=0,1,2, \ldots\right\}$ is a discrete Markov chain with the one-step transition probability matrix $\mathrm{P}(h)=\left(\mathrm{P}_{i j}(h)\right)_{N \times N}=e^{h \Gamma}$.

Since the $\gamma_{i j}$ are independent of $x$, the paths of Markov chain $r$ can be generated independent of $x$ and, in fact, before computing $x$.

For the purpose of stability, assume that $f(0,0,0, i)=g(0,0,0, i)=0$.This shows that $(1.1)$ admits a trivial solution. We assume that $f$ and $g$ satisfy the local Lipschitz condition: 
$\left(\mathrm{H}_{1}\right)$ : Both $f$ and $g$ satisfy the local Lipschitz condition. That is, for each $k=1,2, \ldots$, there is an $h_{k}>0$ such that

$$
|f(x, y, t, i)-f(\bar{x}, \bar{y}, t, i)|+|g(x, y, t, i)-g(\bar{x}, \bar{y}, t, i)| \leq h_{k}(|x-\bar{x}|+|y-\bar{y}|)
$$

for all $t \geq 0$ and $i \in S$ and those $x, y, \bar{x}, \bar{y} \in \mathrm{R}^{d}$ with $|x| \vee|y| \vee|\bar{x}| \vee|\bar{y}| \leq k$.

$\left(\mathrm{H}_{2}\right)$ : For every $i \in S$, there are constants $a_{i} \in \mathrm{R}$ nonnegative constants $\mu_{i}, \sigma_{i}$ and $\beta_{i}$ such that

$$
\begin{gathered}
2[x-N(y)]^{\mathrm{T}} f(x, y, t, i) \leq-a_{i}|x|^{2}+\mu_{i}|y|^{2}, \\
|g(x, y, t, i)|^{2} \leq \sigma_{i}|x|^{2}+\beta_{i}|y|^{2},
\end{gathered}
$$

for all $x, y \in \mathrm{R}^{d}$ and $t \geq 0$.

We also assume that: exists a positive constant $0<\lambda<\frac{1}{2}$, such that $|N| \leq \lambda$.

Considering equation (1.1), we can match in the following form:

$$
f(x(t), x(t-\tau), t, r(t))=-A(r(t)) x(t)+B(r(t)) x(t-\tau)
$$

and $g(x(t), x(t-\tau), t, r(t))=D(r(t)) x(t)+E(r(t)) x(t-\tau)$.

If we take $a_{i}=(\lambda-2) A(i)+|B(i)|, \mu_{i}=|A(i)| \lambda+|B(i)|, \sigma_{i}=|D(i)|, \beta_{i}=|E(i)|$, then the hypotheses $\left(\mathrm{H}_{1}\right)$ and $\left(\mathrm{H}_{2}\right)$ are satisfied naturally for Eq.(1.1). Then for any given initial data $\varphi \in C_{F_{0}}^{b}\left([-\tau, 0], \mathrm{R}^{d}\right)$. Eq. (1.1) has a unique continuous solution $x(t)$.

\section{Math Stability Analysis of the BE Method}

Applying the Backward Euler (BE) method to the linear neutral delay differential system with Markovian switching (1.1) leading to a numerical process of the following type:

$$
x_{n+1}-N x_{n+1-m}=x_{n}-N x_{n-m}+\left(-A\left(r_{n+1}^{h}\right) x_{n+1}+B\left(r_{n+1}^{h}\right) x_{n+1-m}\right) h+\left(D\left(r_{n}^{h}\right) x_{n}+E\left(r_{n}^{h}\right) x_{n-m}\right) \Delta w_{n},
$$

where $h>0$ is a stepsize which satisfies $\tau=m h$ for some positive integer $m$ and $t_{n}=n h, r_{n}^{h}=r\left(t_{n}\right) \in S$, $x_{n}$ is an approximation to $x\left(t_{n}\right)=x_{n}$, if $t_{n} \leq 0$, we have $x_{n}=\varphi\left(t_{n}\right)$, the increments $\Delta w_{k}=w\left(t_{k+1}\right)-w\left(t_{k}\right)$, $k=0,1,2, \ldots$, are independent $N(0, h)$ - distributed

Gaussian random variables. Further, we assume that $x_{n}$ is $\mathrm{F}_{t_{n}}$-measurable at the mesh points $t_{n}$.

Definition 3.1 A numerical method is said to be exponential mean square stable, if there exists a $h_{0}\left(A_{i}, B_{i}, D_{i}, E_{i}\right)>0$ and positive constant $\gamma$, such that, for $h \in\left(0, h_{0}\right)$ with $h=\frac{\tau}{m}$, any application of the method to problem (1.1) generates a numerical approximation $\left\{x_{k}\right\}$, which satisfies

$$
\lim _{t \rightarrow \infty} \sup \frac{1}{t} \log \mathrm{E}\left|x_{k}\right|^{2} \leq-\frac{\gamma}{2}
$$


Theorem 3.1 Assume that there are four nonnegative constants $a_{i}, \mu_{i}, \sigma_{i}, \beta_{i}$, such that conditions (2.2) -( 2.4) hold, and for any $i \in S, 3 A_{i}+\left|D_{i}\right|+\left|E_{i}\right|<0$, where $A_{i}=A(i), D_{i}=D(i)$ and $E_{i}=E(i)$. Then the BE method is exponential mean square stable.

Proof. Let $Z_{k}=x_{k}-N x_{k-m}$, then from conditions (2.2)-(2.4), we have

$$
\begin{aligned}
\left|Z_{k+1}\right|^{2} & =\left\langle Z_{k+1}, Z_{k}+f\left(x_{k+1}, x_{k+1-m}, t_{k+1}, i\right) h+g\left(x_{k}, x_{k-m}, t_{k}, i\right) \Delta w_{k}\right\rangle \\
& =Z_{k+1}{ }^{\mathrm{T}} f\left(x_{k+1}, x_{k+1-m}, t_{k+1}, i\right) h+\left\langle Z_{k+1}, Z_{k}+g\left(x_{k}, x_{k-m}, t_{k}, i\right) \Delta w_{k}\right\rangle \\
& \leq \frac{1}{2}\left(-a_{i}\left|x_{k+1}\right|^{2}+\mu_{i}\left|x_{k+1-m}\right|^{2}\right) h+\frac{1}{2}\left[\left|Z_{k+1}\right|^{2}+\left|Z_{k}\right|^{2}+\left(\sigma_{i}\left|x_{k}\right|^{2}+\beta_{i}\left|x_{k-m}\right|^{2}\right) h\right]+\frac{1}{2} m_{k}^{h}
\end{aligned}
$$

where $\quad m_{k}^{h}=\left|g\left(x_{k}, x_{k-m}, k h, i\right)\right|^{2}\left(\left|\Delta w_{k}\right|^{2}-h\right)+2 Z_{k}^{\mathrm{T}} g\left(x_{k}, x_{k-m}, k h, i\right) \Delta w_{k}$.

Then we have

$$
\left|Z_{k+1}\right|^{2} \leq\left|Z_{k}\right|^{2}+\left(-a_{i}\left|x_{k+1}\right|^{2}+\mu_{i}\left|x_{k+1-m}\right|^{2}\right) h+\left(\sigma_{i}\left|x_{k}\right|^{2}+\beta_{i}\left|x_{k-m}\right|^{2}\right) h+m_{k}^{h}
$$

Note that $\mathrm{E} m_{k}^{h}=0$ for all $k>0$.Taking expectation on the both sides of inequality (3.3), we have

$$
\mathrm{E}\left|Z_{k+1}\right|^{2} \leq \mathrm{E}\left|Z_{k}\right|^{2}+\left(-a_{i} \mathrm{E}\left|x_{k+1}\right|^{2}+\mu_{i} \mathrm{E}\left|x_{k+1-m}\right|^{2}\right) h+\left(\sigma_{i} \mathrm{E}\left|x_{k}\right|^{2}+\beta_{i}\left|x_{k-m}\right|^{2}\right) h
$$

For any $C>1$, we have

$$
\begin{aligned}
{\left[C^{(k+1) h} \mathrm{E}\left|Z_{k+1}\right|^{2}-C^{k h} \mathrm{E}\left|Z_{k}\right|^{2}\right] } & \leq\left(C^{(k+1) h}-C^{k h}\right) \mathrm{E}\left|Z_{k}\right|^{2}+\left(-a_{i} \mathrm{E}\left|x_{k+1}\right|^{2}+\mu_{i} \mathrm{E}\left|x_{k+1-m}\right|^{2}\right) h C^{(k+1) h} \\
& +\left(\sigma_{i} \mathrm{E}\left|x_{k}\right|^{2}+\beta_{i} \mathrm{E}\left|x_{k-m}\right|^{2}\right) h C^{(k+1) h}
\end{aligned}
$$

which implies

$$
\begin{aligned}
C^{k h} \mathrm{E}\left|Z_{k}\right|^{2} \leq & \mathrm{E}\left|Z_{0}\right|^{2}-a_{i} h \sum_{j=0}^{k-1} C^{(j+1) h} \mathrm{E}\left|x_{j+1}\right|^{2}+\mu_{i} h \sum_{j=0}^{k-1} C^{(j+1) h} \mathrm{E}\left|x_{j+1-m}\right|^{2} \\
& +\left[\sigma_{i} h C^{h}+\left(1+\varepsilon_{0}\right)\left(C^{h}-1\right)\right] \sum_{j=0}^{k-1} C^{j h} \mathrm{E}\left|x_{j}\right|^{2} \\
& +\left[\beta_{i} h C^{h}+\lambda^{2}\left(1+\varepsilon_{0}^{-1}\right)\left(C^{h}-1\right)\right] \sum_{j=0}^{k-1} C^{j h} \mathrm{E}\left|x_{j-m}\right|^{2}
\end{aligned}
$$

where we used the inequality $\left|Z_{j}\right|^{2}=\left|x_{j}-N x_{j-m}\right|^{2} \leq\left(1+\varepsilon_{0}\right)\left(\left|x_{j}\right|^{2}+\frac{\lambda^{2}}{\varepsilon_{0}}\left|x_{j-m}\right|^{2}\right)$.

Note that

$$
\begin{aligned}
\sum_{j=0}^{k-1} C^{(j+1) h} \mathrm{E}\left|x_{j+1}\right|^{2} & =\sum_{j=1}^{k} C^{j h} \mathrm{E}\left|x_{j}\right|^{2} \\
& =\sum_{j=0}^{k-1} C^{j h} \mathrm{E}\left|x_{j}\right|^{2}-\mathrm{E}\left|x_{0}\right|^{2}+C^{k h} \mathrm{E}\left|x_{k}\right|^{2}
\end{aligned}
$$




$$
\begin{aligned}
\sum_{j=0}^{k-1} C^{(j+1) h} \mathrm{E}\left|x_{j+1-m}\right|^{2} & =\sum_{j=-m+1}^{k-m} C^{(j+m) h} \mathrm{E}\left|x_{j}\right|^{2} \\
& =C^{m h} \sum_{j=-m+1}^{-1} C^{j h} \mathrm{E}\left|x_{j}\right|^{2}+C^{m h} \sum_{j=0}^{k-1} C^{j h} \mathrm{E}\left|x_{j}\right|^{2}-C^{m h} \sum_{j=k-m+1}^{k-1} C^{j h} \mathrm{E}\left|x_{j}\right|^{2}
\end{aligned}
$$

and

$$
\begin{aligned}
\sum_{i=0}^{k-1} C^{i h} \mathrm{E}\left|x_{i-m}\right|^{2} & =\sum_{i=-m}^{k-m-1} C^{(i+m) h} \mathrm{E}\left|x_{i}\right|^{2} \\
& =C^{m h} \sum_{i=-m}^{-1} C^{i h} \mathrm{E}\left|x_{i}\right|^{2}+C^{m h} \sum_{i=0}^{k-1} C^{i h} \mathrm{E}\left|x_{i}\right|^{2}-C^{m h} \sum_{i=k-m}^{k-1} C^{i h} \mathrm{E}\left|x_{i}\right|^{2}
\end{aligned}
$$

We therefore have

$$
C^{k h} \mathrm{E}\left|Z_{k}\right|^{2} \leq X+\mathrm{E}\left|Z_{0}\right|^{2}+a_{i} h \mathrm{E}\left|x_{0}\right|^{2}+\Delta(C) h \sum_{i=0}^{k-1} C^{i h} \mathrm{E}\left|x_{i}\right|^{2}
$$

where

$$
\begin{gathered}
X=\left[\mu_{i}+\beta_{i} C^{h}+\lambda^{2}\left(1+\varepsilon_{0}^{-1}\right)\left(C^{h}-1\right) / h\right] C^{\tau} h \sum_{i=-m+1}^{-1} C^{i h} \mathrm{E}\left|x_{i}\right|^{2}, \\
\Delta(C)=-a_{i}+\mu_{i} C^{\tau}+\sigma_{i} C^{h}+\left(1+\varepsilon_{0}\right) \frac{C^{h}-1}{h}+\left[\beta_{i} C^{h}+\lambda^{2}\left(1+\varepsilon_{0}^{-1}\right) \frac{C^{h}-1}{h}\right] C^{\tau} .
\end{gathered}
$$

It is easy to see that $\Delta\left(\frac{a_{i} \frac{1}{\tau}}{\mu_{i}}\right)>0, \Delta(1)=-a_{i}+\sigma_{i}+\mu_{i}+\beta_{i} \leq 3 A_{i}+\left|D_{i}\right|+\left|E_{i}\right|<0$ and $\Delta^{\prime}(C)>0$ for any $C \geq 1$, hence, there exists unique constant $C_{h}^{*} \in\left(1,{\frac{a_{i}}{\mu_{i}}}^{\frac{1}{\tau}}\right)$ such that $\Delta\left(C_{h}^{*}\right)=0$.

Thus (3.5) yields

$$
C_{h}^{* k h} \mathrm{E}\left|Z_{k}\right|^{2} \leq X+\mathrm{E}\left|Z_{0}\right|^{2}+a_{i} h \mathrm{E}\left|x_{0}\right|^{2} .
$$

In view of (3.6), for any bounded initial condition $\varphi, X$ is nonnegative $\mathrm{F}_{0}$-measurable random variable, thus the right-hand side of (3.7) converges to a finite random variable as $k \rightarrow+\infty$, that is,

$$
\lim _{k \rightarrow \infty} \sup C_{h}^{* k h} \mathrm{E}\left|Z_{k}\right|^{2} \leq \lim _{k \rightarrow \infty} \sup \left(X+\mathrm{E}\left|Z_{0}\right|^{2}+a_{i} h \mathrm{E}\left|x_{0}\right|^{2}\right)<\infty
$$

Define $u=\log C$, then $\delta \in(0, \bar{\varepsilon} / 2)$, and we also define $\lim _{h \rightarrow o} u_{h}^{*}=\bar{\varepsilon}$. 
So, for any $\delta \in(0, \bar{\varepsilon} / 2)$, there exists $h_{0}=h_{0}\left(A_{i}, B_{i}, D_{i}, E_{i}\right)$ which is the solution of $\Delta\left(C_{h}^{*}\right)=0$, such that for any $h \in\left(0, h_{0}\right), u_{h}^{*}>\bar{\varepsilon}-2 \delta$.

Since we earlier obtained that $\lim _{k \rightarrow \infty} \sup _{h}^{* k h} \mathrm{E}\left|Z_{k}\right|^{2}<\infty$, so we get, for any $h \in\left(0, h_{0}\right)$, that

$$
\bar{\sigma}=\lim _{k \rightarrow \infty} \sup e^{(\varepsilon-2 \delta)^{k h}} \mathrm{E}\left|Z_{k}\right|^{2}<\infty
$$

Consequently, for any $\gamma \in\left(0, e^{-\frac{1}{\tau} \log \lambda \wedge(\bar{\varepsilon}-2 \delta)}\right)$, there exists an integer $k_{1}$, such that $e^{\gamma^{k h}} \mathrm{E}\left|Z_{k}\right|^{2} \leq \bar{\sigma}+\gamma, k \geq k_{1}$.

Applying the elementary inequality $(a+b)^{p} \leq(1+c)^{p-1}\left(a^{p}+c^{1-p} b^{p}\right), a, b>0, p>1, c>0$, with $p=2$ and $c=\lambda /(1-\lambda)$, as well as the condition (2.4), we obtain

$$
e^{\gamma^{k h}} \mathrm{E}\left|x_{k}\right|^{2} \leq(1-\lambda)^{-1} e^{\gamma^{k h}} \mathrm{E}\left|Z_{k}\right|^{2}+\lambda e^{\gamma^{k h}} \mathrm{E}\left|x_{k-m}\right|^{2},
$$

then, for any integer $k_{2}>k_{1}$,

$$
\begin{gathered}
\sup _{k_{1} \leq k \leq k_{2}} e^{\gamma^{k h}} \mathrm{E}\left|x_{k}\right|^{2} \leq(1-\lambda)^{-1}(\bar{\sigma}+\gamma)+\lambda \sup _{k_{1} \leq k \leq k_{2}} e^{\gamma^{k h}} \mathrm{E}\left|x_{k-m}\right|^{2} \leq(1-\lambda)^{-1}(\bar{\sigma}+\gamma)+\lambda e^{\gamma^{\tau}} \sup _{k_{1} \leq k \leq k_{2}} e^{\gamma^{(k-m) h} \mathrm{E}\left|x_{k-m}\right|^{2}} \\
\leq(1-\lambda)^{-1}(\bar{\sigma}+\gamma)+\lambda e^{\gamma^{\tau}} \sup _{k_{1}-m \leq k \leq k_{1}} e^{\gamma^{k h}} \mathrm{E}\left|x_{k}\right|^{2}+\lambda e^{\gamma^{\tau}} \sup _{k_{1} \leq k \leq k_{2}} e^{\gamma^{k h}} \mathrm{E}\left|x_{k}\right|^{2}
\end{gathered}
$$

implying that

$$
\sup _{k_{1} \leq k \leq k_{2}} e^{\gamma^{k h}} \mathrm{E}\left|x_{k}\right|^{2} \leq\left(1-\lambda e^{\gamma^{\tau}}\right)^{-1}\left[(1-\lambda)^{-1}(\bar{\sigma}+\gamma)+\lambda e^{\gamma^{\tau}} \sup _{k_{1}-m \leq k \leq k_{1}} e^{\gamma^{k h}} \mathrm{E}\left|x_{k}\right|^{2}\right] \text {. }
$$

$$
\begin{aligned}
& \text { Letting } k_{2} \rightarrow+\infty \quad \text { in } \\
& \sup _{k_{1} \leq k \leq+\infty} e^{\gamma^{k h}} \mathrm{E}\left|x_{k}\right|^{2} \leq\left(1-\lambda e^{\gamma^{\tau}}\right)^{-1}\left[(1-\lambda)^{-1}(\bar{\sigma}+\gamma)+\lambda e^{\gamma^{\tau}} \sup _{k_{1}-m \leq k \leq k_{1}} e^{\gamma^{k h}} \mathrm{E}\left|x_{k}\right|^{2}\right]<\infty,
\end{aligned}
$$

which gives $\lim _{k \rightarrow+\infty} e^{\gamma^{k h}} \mathrm{E}\left|x_{k}\right|^{2}<\infty$.

Thus the estimate (3.8) yields

$$
\lim _{k \rightarrow+\infty} \sup e^{\gamma^{k h}} \mathrm{E}\left|x_{k}\right|^{2} \leq(1-\lambda)^{-1}(\bar{\sigma}+\gamma)+\lambda e^{\gamma^{\tau}} \lim _{k \rightarrow+\infty} \sup ^{\gamma^{(k-m) h}} \mathrm{E}\left|x_{k-m}\right|^{2},
$$

so, foe any $\gamma \in\left(0, e^{-\frac{1}{\tau} \log \lambda \wedge(\bar{\varepsilon}-2 \delta)}\right)$, we obtain $\lim _{k \rightarrow+\infty} \sup e^{\gamma^{k h}} \mathrm{E}\left|x_{k}\right|^{2} \leq\left(1-\lambda e^{\gamma^{\tau}}\right)^{-1}(1-\lambda)^{-1}(\bar{\sigma}+\gamma)$.

Consequently, $\quad 0=\lim _{k \rightarrow+\infty} \sup \frac{\log e^{\gamma^{k h}} \mathrm{E}\left|x_{k}\right|^{2}}{k h}=\gamma+2 \lim _{k \rightarrow+\infty} \sup \frac{\log \mathrm{E}\left|x_{k}\right|}{k h}$, which gives (3.2).

\section{Numerical Example}


We shall discuss an example to illustrate our theory. Let $w(t)$ be a scalar Brownian motion. Let $r(t)$ be a right continuous Markov chain taking values in $S=\{1,2\}$ with the generator $\Gamma=\left(\gamma_{i j}\right)_{2 \times 2}=\left(\begin{array}{cc}-1 & 1 \\ \gamma & -\gamma\end{array}\right)$.

We assume $w(t)$ and $r(t)$ are independent.

Consider the following one-dimensional linear neutral stochastic delay differential equation with Markovian switching

$$
\left\{\begin{aligned}
d\left[x(t)-\frac{1}{4}(x(t-1))\right]= & {[A(r(t)) x(t)+B(r(t)) x(t-1)] d t } \\
& +[D(r(t)) x(t)+E(r(t)) x(t-1)] d w(t), t \geq 0, \\
x(t)=t+1, t \in[-1,0] &
\end{aligned}\right.
$$

Let $A(1)=-2, B(1)=1, D(1)=0, E(1)=\frac{2}{5}$ and $A(2)=-9, B(2)=4, D(2)=0, E(2)=\frac{4}{5}$.

It is interesting to regard Eq. (4.1) as the result of the following two equations:

$$
d\left[x(t)-\frac{1}{4}(x(t-1))\right]=[-2 x(t)+x(t-1)] d t+\frac{2}{5} x(t-1) d w(t)
$$

and

$$
d\left[x(t)-\frac{1}{4}(x(t-1))\right]=[-9 x(t)+4(t-1)] d t+\frac{4}{5} x(t-1) d w(t)
$$

switching from one to the other according to the movement of the Markov chain $r(t)$. It is known that Eqs. (4.2) and (4.3) are exponentially mean square stable, where $\lambda=\frac{1}{4}, a_{1}=\frac{9}{2}, u_{1}=3, \sigma_{1}=0, \beta_{1}=\frac{2}{5}, a_{2}=\frac{79}{4}, u_{2}=\frac{25}{4}$, $\sigma_{2}=0, \beta_{2}=\frac{4}{5}, 0<\gamma<4$, we take $\gamma=1, h_{0}=h_{0}\left(A_{1}, B_{1}, D_{1}, E_{1}\right)<\frac{1}{11}, h_{0}=h_{0}\left(A_{2}, B_{2}, D_{2}, E_{2}\right)<\frac{1}{9}$.

Thus, when a method of form (3.1) with stepsize $h \in\left(0, \frac{1}{11}\right)$ is used to solve the above system, the corresponding numerical solution is exponential mean-square stable by Theorem 3.1.

\section{Acknowledgment}

This work was supported by the Natural Science Foundation of Heilongjiang Province (A201418) and the Creative Talent Project Foundation of Heilongjiang Province Education Department (UNPYSCT-2015102)

\section{References}

[1] Abolnikov, L., Dshalalow, J. H., \& Treerattrakoon, A., (2008). On a dual hybrid queueing system. Nonlinear Analysis: Hybrid Systems, 2, 96-109.

[2] Matasov, A., \& Piunovkiy, A. B., et al. (2000). Stochastic differential delay equations with Markovian switching. Bernoulli, 6, 73-90.

[3] Xuerong, M. (2000). Robustness of stability of stochastic differential delay equations with Markovian switching. SACTA, 3, 48-61.

[4] Yuan, C., et al. (2006). Stochastic Differential Equations with Markovian Switching. London: Imperial College Press.

[5] Rathinasamy, A., \& Balachandran, K. (2008). Mean-square stability of second-order Runge-Kutta methods for multi-dimensional linear stochastic differential systems. Journal of Computational and Applied Mathematics, 219,170-197. 
[6] Ronghua, L., \& Yingmin, H. (2006). Convergence and stability of numerical solutions to SDDEs with Markovian switching. Applied Mathematics and Computation, 175, 1080-1091.

[7] Rathinasamy, A., \& Balachandran, K. (2008). Mean-square stability of Milstein method for linear hybrid stochastic delay integro-differential equations. Nonlinear Analysis: Hybrid Systems, 2, 1256-1263.

[8] Xuerong, M. (1995). Exponential stability in mean square of neutral stochastic differential functional equations. Systems \&Control Letters, 26, 245-251.

[9] Cong, S. (2013). On exponential stability conditions of linear neutral stochastic differential systems with time-varying delay. International Journal of Robust and Nonlinear Control, 23(11), 1265-1276.

[10] Lirong, H., \& Xuerong, M. (2009). Delay-dependent exponential stability of neutral stochastic delay systems. IEEE Transactions on Astomatic Control, 54,147-152.

[11] Gouaisbaut, F., \& Peaucelle, D. (2006). Delay-dependent stability analysis of linear time delay systems. IFAC workshop on Time Delay System, 6(1), 54-59.

[12] Fuke, W., \& Chengming, H. (2015). Exponential mean square stability of the theta approximations for neutral stochastic differential delay equations. Journal of Computational and Applied Mathematics, 286, 172-185.

[13] Wansheng, W. (2011). Mean-square stability of semi-implicit Euler method for nonlinear neutral stochastic delay differential equations. Applied Numerical Mathematics, 61, 696-701.

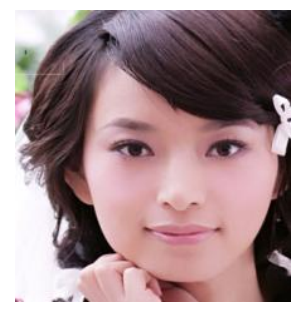

Yuan Haiyan was born in April 1982. She got the PhD of HIT and the postdoctor of Harbin Engineering University. Her major interesting is the study of mathematics and applied mathematics. She is also interested in the optimization algorithm and system modeling in research work.

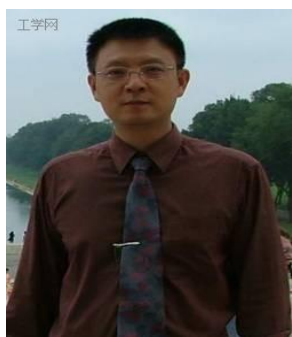

Shen Jihong was born in September 1966. He got the doctor of engineering. He is a professor, Ph.D. supervisor. He is the dean of College of science, Harbin Engineering University. His major interesting is the study of mathematics and applied mathematics. He is also interested in the optimization algorithm and system modeling in research work. 